\title{
Combining the High Tech with the Soft Touch: Population Health Management Using eHealth and Peer Support
}

\author{
Sarah D. Kowitt, MPH, ${ }^{1}$ Patrick Y. Tang, MPH, ${ }^{1}$ Malinda Peeples, MS, RN,2 Janet Duni, MPA, RN, CCM, \\ Steven Peskin, MD, MBA, FACP, and Edwin B. Fisher, $\mathrm{PHD}^{1}$
}

\section{Background}

I NTEGRATION OF DIVERSE approaches may offer paths to meeting population health challenges, such as how to provide ongoing diabetes self-management support to the 387 million people with the disease around the world. ${ }^{1}$ The Affordable Care Act challenges us to improve the health of whole populations, with an eye toward chronic diseases in particular. The centrality of highly variable human behavior provides enormous challenges to managing populations of those with chronic diseases. It requires reorienting resources within the health care system to address ongoing, effortful, and complex behaviors of patients amid strong competing priorities.

Major reviews ${ }^{2}$ document that peer support provided by "community health workers," "lay health advisors," "promotores," "patient navigators," and individuals with a number of other titles can make important contributions to health care and prevention, including chronic care. However, many studies focus on selected, sometimes high-risk samples or samples of special interest. As much as these may illuminate effectiveness and key features of peer support, they provide little guidance as to how to take peer support to scale to serve the populations who may benefit from it. For example, to serve the estimated 387 million with diabetes worldwide would require 715,000 full-time peer supporters, each with caseloads of 500 , or 39 million volunteers, each assisting 10 patients.

Although sometimes seen as antithetical to the person-toperson contact of peer support, the spread of the Internet, social media, and smartphones, and the arrival of each technological advance offer new ways to deliver health care services, including those to support prevention and ongoing disease management. In the past 2 decades, many studies have affirmed the effectiveness of eHealth for a variety of health outcomes. ${ }^{3}$ Although eHealth offers a platform for reaching populations, we cannot rely on technology alone. In their current state, consumer eHealth technologies sometimes have difficulty engaging people and cannot comprehensively address people's complex needs. ${ }^{4}$

In contrast to the eHealth model, peer support delivers highly personalized and engaging support to people at all levels of need. ${ }^{5}$ Importantly, this includes the ability of peer support to (1) reach and engage those who are often "hardly reached" by clinical and preventive services, and (2) reduce emotional and psychosocial distress among those with chronic conditions and the avoidable acute and hospital care that so often accompany it. ${ }^{5}$ Studies have documented the effectiveness, feasibility, and wide acceptability of peer support for chronic disease management and prevention in diverse settings and populations.,

With the engagement of peer support and the reach of eHealth (and the effectiveness and feasibility of both approaches), we therefore ask: Can we integrate the high tech of eHealth with the soft touch of peer support to manage the health of populations? If so, what might such an integration look like?

\section{Our Model}

Peers for Progress, with support from the Gillings Innovation Lab program at the University of North Carolina at Chapel Hill, is developing and testing a model that integrates eHealth with lay health coaching for people living with type 2 diabetes. The base of the program will be a one-toone relationship of the patient with a health coach. WellDoc's BlueStar diabetes "app," 6 the first Food and Drug Administration-cleared mobile prescription therapy (accessible on both smartphones and computers) will deliver diabetes education, monitoring, and messaging and so reduce the

\footnotetext{
${ }^{1}$ Peers for Progress and Department of Health Behavior, Gillings School of Global Public Health, University of North Carolina at Chapel Hill, Chapel Hill, North Carolina.

${ }^{2}$ WellDoc, Inc., Baltimore, Maryland.

${ }^{3}$ Vanguard Medical Group, Verona, New Jersey.

${ }^{4}$ Horizon-BCBSNJ and Rutgers Robert Wood Johnson Medical School, Newark, New Jersey.
} 
demands on live coaches to deliver such services. The pilot will be implemented in collaboration with Horizon Blue Cross Blue Shield and Vanguard Medical Group, a primary care group practice in north and central New Jersey. Three out of 5 of the Vanguard sites are recognized as patient-centered medical homes with care coordination processes to manage high-risk patients. The pilot test will aim to engage 200 patients referred from Vanguard's clinics.

A key component of our program will be titrating different levels of live peer support according to the needs and preferences of patients. Adults with diabetes with good selfmanagement and motivation may have their needs adequately addressed by the BlueStar app and only occasional health coaching. More intensive coaching may be reserved for patients with greater needs, such as those with poor selfmanagement, complex multimorbidities, or psychosocial concerns. BlueStar's features can handle the more routine tasks of self-management, allowing health coaches to provide more individualized services. Cutting across this titration of services, patients will be free to access BlueStar, the health coach, and/or both, according to their preferences and sense of need.

\section{Formative Evaluation and Protocol Refinement}

In order to tailor intervention components to the needs of all stakeholders involved, we conducted qualitative research with patients, medical providers, and care coordinators at Vanguard. With the help of the director of care coordination, purposive sampling identified and recruited 4 care coordinators (all registered nurses), 1 medical provider (physician assistant), and 4 patients with type 2 diabetes (aged 52 to 80 years; living with diabetes for a range of 7 to 40 years). Semi-structured interviews lasting between 30 and 55 minutes were conducted with each participant to explore their experiences and perspectives regarding diabetes management, eHealth, intervention components, and potential barriers to implementation and dissemination.

These semi-structured interviews revealed widespread interest in the model of integrated peer support and eHealth. All patients said they would benefit from at least 1 element of the intervention (eg, eHealth, peer support) and all care coordinators and the medical provider said the program would be beneficial to both the patients and their practice. Some patients, for instance, said that they would benefit from access to the diabetes app (specifically for its ability to help them record and track blood glucose values), while others said that speaking with a health coach would be most valuable. Participants also differed in the frequency and mode of communication through which they would want to be contacted by health coaches, with some patients preferring more frequent contact over the phone (eg, once or twice a week) and others preferring less frequent contact via e-mail or text messaging. Contrary to the common misperception that older adults are reluctant to engage with eHealth interventions, none of the patients expressed hesitancy about using the app, despite variations in age.

Interviews in the formative evaluation raised several potential challenges for the program. These have led to revisions of the protocols for training the coaches and the actual intervention. First, although, care coordinators described how patients' receptivity to phone calls has improved over the past few years, reaching and engaging some patients by phone can be challenging. Based on feedback from the clinical team, our pilot will thus (1) mail letters from patients' providers introducing the program and health coaches and (2) provide forewarning of phone calls (and area codes that phone calls will be coming from) to patients before initial calls begin.

Second, the medical team raised concerns over the qualifications of the health coaches. We have addressed this in 2 ways: by clarifying the roles of the coaches and by careful training and protocol development. We will emphasize that health coaches will be different from and complementary to clinical providers. Their role will include encouraging patients to gain the clinical care they need (such as in response to emergent issues), "linkage to clinical care" being a key function of the Peers for Progress model of peer support. However, they will not advise patients on treatment choices or objectives. If patients raise questions about specific treatments, the role of the coach will be to encourage patients to pursue such questions with the clinical team.

Training the coaches will ensure that they have sufficient knowledge of diabetes management, especially regarding how patients can make dietary and exercise changes (the most commonly cited barriers to diabetes management among patients in this study). To address these concerns, health coaches will receive substantial training before and continuing during intervention implementation, and be linked with a care coordinator who will be able to provide backup support and referrals for care as needed.

The medical team also emphasized that it was not enough that the coach know about diabetes and its care; the health coach also would need to know what was appropriate for the community Vanguard serves. Thus, even though all coaching will be by phone and could easily be arranged from a remote location, the coaches will be recruited from the communities in which patients reside.

Some patients interviewed as part of the formative evaluation, especially those who have had diabetes for many years (eg, $\geq 25$ years), stated that they either did not need or did not want additional support or resources for their diabetes management. Peers for Progress has identified nondirective, flexible, but persistent approaches to patient recruitment that have been effective; for example, over the course of 2 years, reaching and engaging $89 \%$ of ethnic minority, low-income single mothers of Medicaid-covered children hospitalized for asthma, ${ }^{8}$ or reaching and engaging more than $85 \%$ of all patients with diabetes served through a large, urban Federally Qualified Health Center. ${ }^{5}$ Accordingly, protocols for health coaches will highlight how contact can be made with patients who initially may be unreceptive to receiving support by (1) describing the services available, (2) focusing on tangible barriers to management and answers to questions, following up from previous doctor visits, (3) recognizing and complimenting patients' feelings that they are "doing just fine," (4) not demanding immediate active participation, and (5) recontacting patients several weeks to a month later (with coaches encouraged to use their own judgment in specifying this) in order to "check in with" (not "check up on") them to see if there are ways the coaches might then be helpful to them.

\section{Future Applications}

Most notably, our program operates on the assumption that although peer support and BlueStar independently will 
be beneficial to patients, their synergy is what will make this program successful. A useful way we have thought about the complementarity between eHealth and peer support is by looking at Peers for Progress' 4 key functions framework that delineates the strategies through which interventions work. These 4 key functions were developed as a template for dissemination of live peer support, ${ }^{7}$ but review of eHealth and telehealth interventions (eg, Oldenburg's Telephone-Linked Care for diabetes management in rural Australia $)^{9}$ has clarified how the 4 key functions also can be satisfied using eHealth technologies. ${ }^{5}$ Within eHealth applications such as BlueStar, assistance with daily management is provided through dialogues and individualized messages. Linkage to clinical care can be arranged by monitoring patients' data and linking them with clinical providers when necessary. Once the system is put in place, ongoing support will be maintained with messaging that is responsive to the evolution of patients' needs. Perhaps most surprisingly, eHealth also can meet the substantial social and emotional support needs of patients. For instance, $79 \%$ of the users of Oldenburg and colleagues' telehealth automated messaging system reported that the system gave them confidence to better manage their diabetes. ${ }^{10}$ Peers for Progress sponsored a recent working conference on the integration of eHealth and peer support, the report from which is available at http://peersforprogress.org/wp-content/ uploads/2015/08/150803-high-tech-soft-touch-brief.pdf.

Given what we know about the benefits of peer support and eHealth, the goal of this pilot will be to understand and fine-tune features of the program that can be applied in future to scale up to entire health care systems and populations. Our pilot is unique given the collaboration of 3 partners with varying expertise: Peers for Progress in peer and social support research, WellDoc in the development and implementation of diabetes management eHealth applications, and Vanguard and Horizon Blue Cross Blue Shield with leadership in primary care and health care delivery. Our model could easily be exported to other health care settings and health management organizations. Lessons learned already have and will continue to identify ways to address implementation and dissemination barriers but should result in a scalable model for the major population health challenge of providing diabetes self-management support for entire populations of those with the disease.

\section{Author Disclosure Statement}

The authors declared the following potential conflicts of interest with respect to the research, authorship, and/or publication of this article: Dr. Fisher, Ms. Kowitt, and Mr. Tang work at Peers for Progress, Ms. Peeples works for WellDoc, Ms. Duni works for Vanguard Medical Group, and Dr. Peskin works for Horizon BCBSNJ_all of which are discussed in this commentary.
This project was supported by a grant from the Gillings Innovation Laboratory, an initiative of the Gillings School of Global Public Health at the University of North CarolinaChapel Hill. The authors received no financial support for this commentary.

\section{References}

1. International Diabetes Federation. IDF Diabetes Atlas. Brussels, Belgium: International Diabetes Federation, 2013.

2. Perry HB, Zulliger R, Rogers MM. Community health workers in low-, middle-, and high-income countries: An overview of their history, recent evolution, and current effectiveness. Ann Rev Public Health 2014;35:399-421.

3. Cotter AP, Durant N, Agne AA, Cherrington AL. Internet interventions to support lifestyle modification for diabetes management: A systematic review of the evidence. J Diabetes Complications 2014;28:243-251.

4. Glueckauf RL, Lustria M. E-health self-care interventions for persons with chronic illnesses: Review and future directions. In: Parker JC, Thorson E, eds. Health Communication in the New Media Landscape. New York: Springer Publishing Company, LLC, 2009:151-164.

5. Fisher EB, Ballesteros J, Bhushan N, et al. Key features of peer support in chronic disease prevention and management. Health Aff 2015;34:1523-1530.

6. Peeples MM, Iyer AK, Cohen JL. Integration of a mobileintegrated therapy with electronic health records: Lessons learned. J Diabetes Sci Technol 2013;7:602-611.

7. Fisher EB, Boothroyd RI, Coufal MM, et al. Peer support for self-management of diabetes improved outcomes in international settings. Health Aff (Millwood) 2012;31:130 139.

8. Fisher EB, Strunk RC, Highstein GR, et al. A randomized controlled evaluation of the effect of community health workers on hospitalization for asthma: The asthma coach. Arch Pediatr Adolesc Med 2009;163:225-232.

9. Williams ED, Bird D, Forbes AW, et al. Randomised controlled trial of an automated, interactive telephone intervention (TLC Diabetes) to improve type 2 diabetes management: Baseline findings and six-month outcomes. BMC Public Health 2012;12:602.

10. Bird D, Oldenburg B, Wootton R, Friedman R. Development and evaluation of an automated telephone system for diabetes self-management. Annals of Behavioral Medicine 2008;35(Suppl):S013.

Address correspondence to: Sarah Kowitt

Department of Health Behavior, Rosenau Hall, CB \#7440

University of North Carolina at Chapel Hill Chapel Hill, North Carolina 27599-7440

E-mail: Kowitt@email.unc.edu 